\title{
Ultra-sensitive refractive index sensor based on extremely simple femtosecond-laser-induced structure
}

\author{
Pengcheng Chen ${ }^{1}$, Xuewen Shu ${ }^{1, *}$, Hanyuan CaO ${ }^{1}$, Kate Sugden ${ }^{2}$ \\ ${ }^{1}$ Wuhan National Laboratory for Optoelectronics \& School of Optical and Electronic Information, Huazhong University of Science and \\ Technology, Wuhan 430074, China \\ ${ }^{2}$ Aston Institute of Photonic Technologies, Aston University, Birmingham B4 7ET, UK \\ *Corresponding author:xshu@hust.edu.cn
}

Received XX Month XXXX; revised XX Month, XXXX; accepted XX Month XXXX; posted XX Month XXXX (Doc. ID XXXXX); published XX Month XXXX

We demonstrate here an extremely simple, compact and robust RI probe sensor based on a femtosecond-laser induced refractive-index-modified-dot (RIMD) fabricated near the end face of a single mode fiber. The RIMD and the fiber end face form a Fabry-Perot interferometer, which is highly sensitive to surrounding RI. The fabrication process of the RIMD involves only one step and takes $\sim 0.1 \mathrm{~s}$, which is extremely short compared with other techniques. The proposed sensor exhibits an ultrahigh sensitivity of $\sim 2523.2 \mathrm{~dB} / \mathrm{RIU}$ at an RI of 1.435, which is 1-2 orders of magnitude higher than that of the existing intensity-modulated RI sensors. Moreover, the proposed sensor has the distinct advantages of compact size $(\sim 50 \mu \mathrm{m})$, easy fabrication and no temperature cross-sensitivity. The advantages of the device make it a promising candidate for applications in designing highly sensitive sensors in biochemical and environmental measurement field.

\section{(C) 2017 Optical Society of America}

OCIS codes: (060.2370) Fiber optics sensors; (230.4000) Microstructure fabrication; (120.3180) Interferometry; (120.2230) Fabry-Perot. (230.1150) All-optical devices.

http://dx.doi.org/10.1364/OL.99.099999

Optical fiber Fabry-Perot interferometers (FPIs) are attractive as RI sensors because they are compact, have high fringe visibility, high RI sensitivity and are simple to fabricate. Various fiber in-line FPI structures have been proposed. Currently, fiber in-line FPIs are mainly manufactured by fusion splicing different types of fibers together, such as photonics crystal fiber[1] and capillary tube[2]. However, these splicing methods have problems such as mode mismatch between the different parts, the splice being easily broken, and it being difficult to achieve good reproducibility due to the manual operation. Meanwhile, the use of expensive specialty fibers increases the cost of the sensors. Another method to fabricate fiber in-line FPI is to pretreat the end surface in advance, for example using chemical etching[3], multiple splicing arcs[4], or by coating a thin film or wafer $[5,6]$. However, these approaches increase the number of manufacturing steps and the fabrication time. Moreover, the above methods have some inherent challenges. For example, it is difficult to predict or control the length of the cavity precisely and it requires a series of complicated fabrication processes such as assembling multiple components together, precise cleaving and correct arc conditions. In addition, many of those FPIs are rather fragile and have poor robustness. Recently, a direct femtosecond laser (fs-laser) or UV laser micromachining method has also been applied to fabricate air-gap cavities to produce a fiber in-line FPI [7-10]. For fs-laser micromachining, the length of the cavity can be controlled precisely but these devices have very poor mechanical properties and are susceptible to interference effects from things such as bending, or need an extra arc discharge to smooth the inner wall of the microchannel, which makes the fabrication process complicated and time-consuming. With UV laser micromachining, however, it is also hard to control the length of the cavity precisely and it requires additional fusion assembly [10].

In this paper, we propose and demonstrate an ultra-sensitive and extremely simple and robust RI probe sensor based on in-fiber refractive-index-modified-dot (RIMD) induced by femtosecond laser. The RIMD and the end-face of the cleaved single mode fiber (SMF) form a composite-cavity FPI. It can simultaneously measure temperature alongside exhibiting ultra-high RI sensitivity of $\sim 2523.2 \mathrm{~dB} / \mathrm{RIU}$ at the refractive index of 1.435 , which is at least 10 times higher than previous reported results. The laser fabrication process takes only about $0.1 \mathrm{~s}$, which lends itself to efficient manufacture. Furthermore, compared with wavelength 
modulated FPIs, our filling-free intensity modulated FPI is more reliable and attractive due to its insensitivity to bend, no temperature cross-sensitivity and no need for expensive wavelength demodulation equipment. Our further analysis and experiments also reveal two ultra-sensitive zones for such a structure, which may have great significance for sensing application.

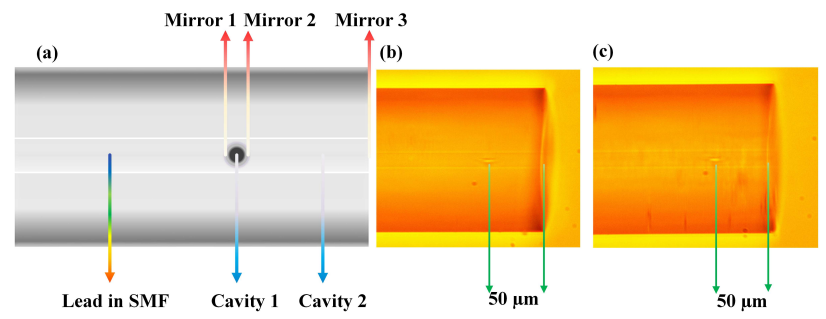

Fig. 1(a) Schematic diagram of the proposed fiber in-line FPI. The top (b) and the side (c) view of microscope images of a FPI sample fabricated by fs laser.

Fig.1(a) shows the schematic diagram of the fiber in-line FPI, which comprises of a well-cleaved SMF and a small RIMD in fiber core. The FPI has a composite cavity formed by two mirrors from the surface of the RIMD (Mirror 1, Mirror 2) and one mirror from the end face of the SMF (Mirror 3).

To fabricate the RIMD, fs laser pulses (520nm, 350fs, $200 \mathrm{kHz}$ ) were focused into the optical fiber through an objective lens (Olympus,40X/0.65). The reflection spectrum of the FPI during fabrication processing can be monitored in real time through a spectral monitoring system, which consists of a broadband light source (BBS), an optical circulator, and an optical spectrum analyzer (OSA, AQ6370) with $0.02 \mathrm{~nm}$ spectral resolution. An online CCD camera monitoring system was employed to record sample images during the fabrication. A standard SMF (YOFC, Ltd.) was used in our experiments.

The fabrication of the device is quite straight-forward. The SMF with a well-cleaved end is first mounted on a highly-precision, computer-controlled 3-D translation stage (Newport, Inc.). After the fiber is positioned correctly, one can open the shutter (with $\sim 100 \mathrm{~ms}$ exposure time) to induce a RIMD in the fiber. The actual fs laser energy used for our fabrication was approximately $1.8 \mu \mathrm{J} / \mathrm{pulse}$. The top and the side view of microscope images of a fabricated device are shown in Fig. 1(b)-(c). The effective diameter of the RIMD was $\sim 4 \mu \mathrm{m}$. The distance between the center of the RIMD and the end face of fiber was $50 \mu \mathrm{m}$.

We investigated the response of the sensor to surrounding RI using sucrose solutions with different concentrations. After each measurement, the sensor head was carefully cleaned with alcohol to eliminate the residual test liquid on the end face of the fiber until to recover its original spectrum in air for next RI test. The response of the FPI probe to the surrounding RI was shown in Fig. 2(a). The free spectrum range (FSR) of the interference fringes is $\sim 16 \mathrm{~nm}$, and thus the corresponding cavity length can be calculated by $L=\lambda^{2} / 2 n_{c o} F S R \approx 52 \mu m$ (the RI of the core $n_{c o}=1.46$ ), which is very close to the designed value. This indicates that the length of the cavity can be predicted and controlled precisely. It can be seen in Fig. 2(a) that the intensity of the reflection spectrum decreases gradually when the RI increases from 1.000 (air), 1.333 (water) to 1.435 (sucrose solution) and it is interesting that the visibility of the interference fringes increases gradually at the same time. The initial visibility is only $\sim 0.8 \mathrm{~dB}$ when the device is in the air. However, when the probe is in the liquid (RI > 1.33), the visibility increases with the RI increasing and it reaches more than $20.7 \mathrm{~dB}$ at the RI of 1.435.
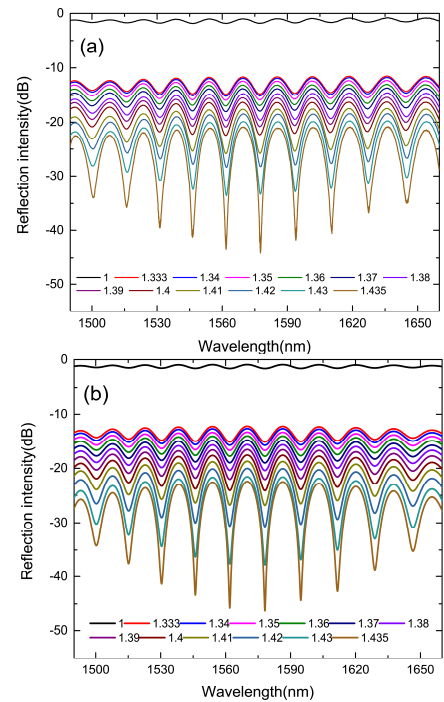

Fig. 2 (a) Reflection spectra of the sensor measured at different RI. (b) Simulated reflection spectrum response at different RI.

The light propagating in the fiber and reflected by the three mirrors will generate a three-wave FPI pattern in fiber. The interference pattern consists of both broad fringes and fine fringes that result from the interference of light inside the composite cavity formed by mirrors 1,2 and 3 . The strength of the interference fringes can be modeled using the following threebeam optical interference equation [10]

$$
\begin{aligned}
I= & R_{1}+A^{2} R_{2}+B^{2} R_{3}-2 \sqrt{R_{1} R_{2}} A \cos \left(2 \varphi_{1}\right)- \\
& 2 \sqrt{R_{1} R_{3}} B \cos \left(2 \varphi_{1}+2 \varphi_{2}\right)+2 A B \sqrt{R_{2} R_{3}} \cos \left(2 \varphi_{2}+\delta(\varphi)\right)
\end{aligned}
$$

Where $A=\left(1-\alpha_{1}\right)\left(1-\gamma_{1}\right)\left(1-R_{1}\right), B=\left(1-\alpha_{1}\right)\left(1-\gamma_{1}\right)\left(1-R_{1}\right)(1$ $\left.-\alpha_{2}\right)\left(1-\gamma_{2}\right)\left(1-R_{2}\right) ; \varphi_{j}=4 \pi n_{j} L_{j} / \lambda$ is the phase shift in the cavity $j(j=1,2) ; n_{j}$ and $L_{j}$ are the refractive index and length of the cavity $j$, respectively; $\alpha_{1}, \alpha_{2}$ are the intensity attenuation factors of the Mirror 1 and Mirror 2; $\gamma_{1}, \gamma_{2}$ are defined as the transmission loss factors of the Cavity 1 and Cavity 2, respectively; $R_{i}$ is the power reflection coefficients at mirror $i(i=1,2,3)$, which can be calculated by the Fresnel formula:

$$
R_{1}=R_{2}=\left(\frac{n_{d o t}-n_{c o}}{n_{d o t}+n_{c o}}\right)^{2}=\left(\frac{\Delta n}{n_{d o t}+n_{c o}}\right)^{2} ; R_{3}=\left(\frac{n_{c o}-n_{e x}}{n_{c o}+n_{e x}}\right)^{2}
$$

Here $n_{d o t}$ is the refractive index of the RIMD, $n_{e x}$ is the RI of the measured liquid. Typically the value of $\Delta n$ induced by fs lasers is $\sim 0.01[11]$.

When $n_{e x}<n_{c o}, \delta(\varphi)=0$. While when $n_{e x}>n_{c o}, \delta(\varphi)=\pi$, there is a $\pi$ phase shift at reflection Mirror 3.

From Eq. (1), the fine fringe visibility $V$ can be derived as: 


$$
V=\frac{I_{\max }-I_{\min }}{I_{\max }+I_{\min }}=\frac{2 \frac{B \sqrt{R_{3}}}{\sqrt{R_{1}}+A \sqrt{R_{2}}}}{1+\left(\frac{B \sqrt{R_{3}}}{\sqrt{R_{1}}+A \sqrt{R_{2}}}\right)^{2}}
$$

From Eq. (2), one can see that only $R_{3}$ depends on the surrounding RI to be measured. When the surrounding medium is air, $\sqrt{R_{1}}+A \sqrt{R_{2}} \ll B \sqrt{R_{3}}$, thus $V \sim 0$, i.e. the visibility of the interference fringe pattern is very low. However, if the surrounding RI is increased, so that $B \sqrt{R_{3}}$ is approaching $\sqrt{R_{1}}+A \sqrt{R_{2}}, V$ will increase to $\sim 1$, which means maximum visibility can be obtained.

In order to illustrate this visually, we can simulate the evolution of the reflection spectra of the RI sensor at various RI based on equations (1) and (2) by calculating the normalized reflection spectrum from $1490 \mathrm{~nm}$ to $1660 \mathrm{~nm}$ with $\alpha_{1}=\alpha_{2}=0.06, \gamma_{1}=\gamma_{2}=$ $0.01, \Delta n=0.01, n_{c o}=1.46$, respectively. The calculated reflection spectra under various surrounding RIs are shown in Fig. 2(b). Comparing Figs. 2(a) and (b), it is clearly seen that the simulated spectra are in good agreement with the experimental spectra.
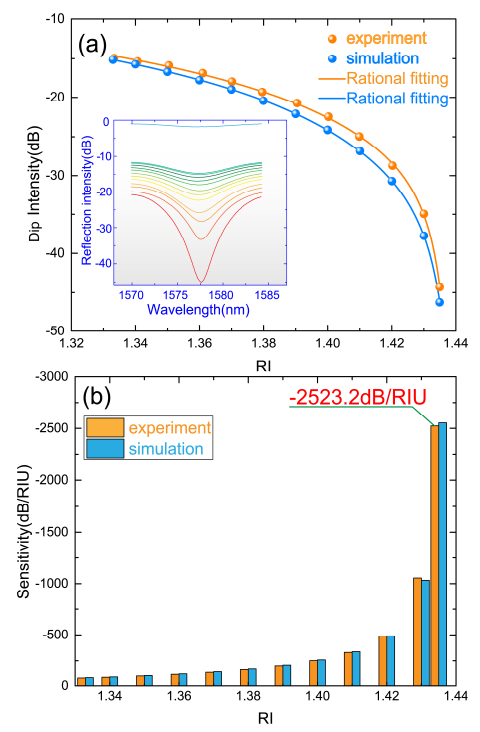

Fig. 3 (a) Measured and simulated fringe dip intensity at 1578nm versus surrounding RI and their rational fitting curves. (b) Measured and simulated RI sensitivity at different RI.

The strength of the dip of any of the fringes can be used as a RI indicator. Fig. 3(a) shows the strength of the $1578 \mathrm{~m}$ dip as a function of the surrounding RI for both experimental measurement and the simulation. It is noted in Fig. 3(a) that the dip intensity of the interference fringe decreases rapidly with the increase of the surrounding RI. The fitting curves adopted in Fig. 3(a) use rational fitting $\left((a x+b) /\left(c x^{2}+d x+e\right)\right)$, and have a correlation coefficient $\left(\mathrm{R}^{2}\right)$ of 0.9995 and 0.9999 for the experimental data and the simulated data, respectively. It is clearly seen that the trends of the response of the dip strength to the surrounding RI in the experiment and the simulation agree very well. Fig. 3(b) presents the comparison of the RI sensitivities measured in the experiment and the ones calculated in the simulation. It is obvious that the experimental results exhibit high consistency with the simulated results. The sensitivity of the fringe dip intensity to the surrounding RI is found to be $\sim 2523.3 \mathrm{~dB} / \mathrm{RIU}$ at the RI of 1.435 . Given $0.001 \mathrm{~dB}$ power resolution of the OSA, an ultra-high RI resolution of $4.0 \times 10^{-7} \mathrm{RIU}$ could be achieved at the RI of 1.435 . To our best knowledge, this is the highest RI resolution and also the highest RI sensitivity obtained for RI sensors based on intensity measurement to date. Table 1 compares our RI sensor with the existing fiber-optic RI sensors based on intensity modulation.

Table 1. Comparison of the existing optical fiber intensitymodulated RI sensors

\begin{tabular}{lll}
\hline $\begin{array}{l}\text { Sensor } \\
\text { Structure }\end{array}$ & $\begin{array}{l}\text { Fabrication } \\
\text { Technique }\end{array}$ & $\begin{array}{l}\text { RI Sensitivity } \\
\text { (dB/RIU) }\end{array}$ \\
\hline TCF-MI[12] & Special splice & Max. 202.5 \\
MMF- MMI[13] & Special splice & Max. 87 \\
FBG- FPI [14] & UV PMSBT. & $\sim 1$ \\
PCF- FPI [1] & Special splice & $\sim 11.5$ \\
HOF- FPI[2] & Special splice & 16 \\
Air Gap-FPI[15] & HF Acid Etching & 29.7 \\
Air Gap-FPI[10] & UV Laser M.mac. & 24 \\
Air hole[16] & Fs M.mac. & Max. 110 \\
\hline
\end{tabular}

RIMD-FPI[our sensor] $\quad$ One Step Fs M.mac. Max. 2523.3

MI: Michelson interference; MMI: Multimode Interference; FPI: Fabry Perot interferometer; M.mac: Micro-machining PMSBT: phase mask-scanning beam technique; Max.: Maximum Value

More interestingly, we find two extremely sensitive zones for RI sensing where $V \sim 1$. To explore this, Fig. 4 shows the relationship between the effective reflectance (i.e. $\sqrt{R_{1}}+A \sqrt{R_{2}}$ and $B \sqrt{R_{3}}$ ) versus surrounding RI. It can be seen that there are two intersection points between the red curve $\left(B \sqrt{R_{3}}\right)$ and the straight blue line $\left(\sqrt{R_{1}}+A \sqrt{R_{2}}\right)$. The RI values for the two intersection points are 1.438 and 1.482 , respectively. We can define four different regions (i.e. $\mathrm{RI}<1.438,1.438<\mathrm{RI}<1.46$, $1.46<\mathrm{RI}<1.482$, and $\mathrm{RI}>1.482$, marked as regions I-IV, respectively), which have different response trends for RI sensing. In order to verify the existence of these two extremely sensitive zones for RI sensing predicted by analysis, we experimentally tested the proposed sensor under higher surrounding RI. The close-up of the measured reflection spectra in regions II - IV for various RI values are shown in Figs. 5(a)-(c), respectively. It is clearly seen the spectral response trends agree well with the ones predicted. It is also experimentally verified the existence of the $\pi$ phase shift due to the half-wave loss. Fig. 5(d) shows the variation of the measured intensity of the interference fringe dip at wavelength 1578nm (for $\mathrm{RI}<1.46$ ) or $1570 \mathrm{~nm}$ (for $\mathrm{RI}>1.46$ ) as a function of the surrounding RI. And the measured intensity changes rapidly near the RI of 1.438 and 1.482 , which means two ultra-sensitive zones are experimentally verified. The ultrasensitive zones provide a highly-sensitive measurement for RI sensing and should find some important applications in practical environmental, chemical and biochemical sensing applications. 


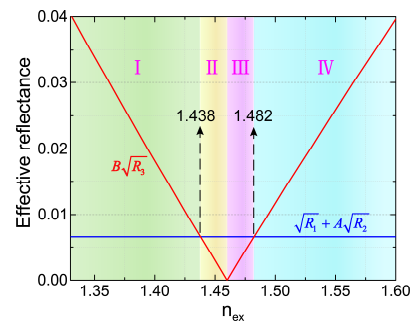

Fig. 4 Variation of the effective reflectance with the surroundings RI.
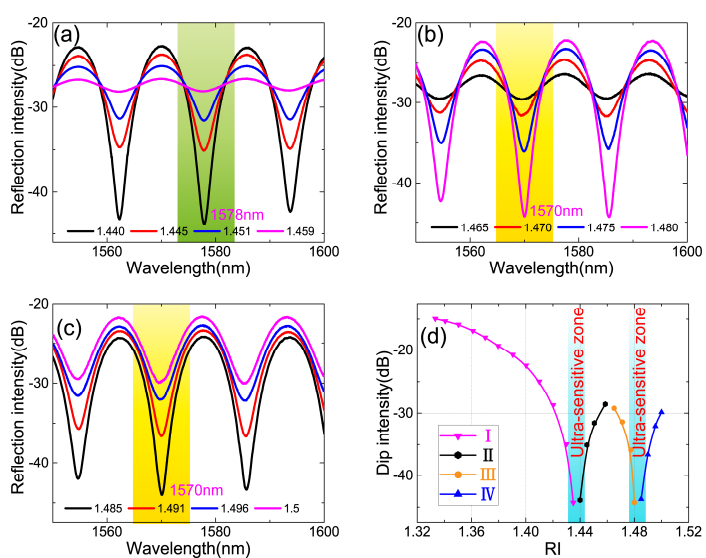

Fig. 5 (a)-(c) Measured reflective spectra of FPI in regions II - IV. (d) Measured fringe dip intensity in four regions versus RI.

We further investigated the temperature response of the proposed RI sensor by mounting it on a hotplate. With increasing temperature, the reflection spectrum of the sensor hardly changed except for the whole spectrum slightly shifting to a longer wavelength. Fig. 6 shows the temperature response of the dip at $\sim 1578 \mathrm{~nm}$, whose spectra was measured during the heating process is also illustrated in the inset. It is clearly seen that the wavelength of the sensor shifted toward a longer wavelength as temperature increased (with a sensitivity of $11.21 \mathrm{pm} /{ }^{\circ} \mathrm{C}$ ), while the dip intensity of the interference fringe hardly changed $(<0.02 \mathrm{~dB})$, indicating that the dip intensity of the interference

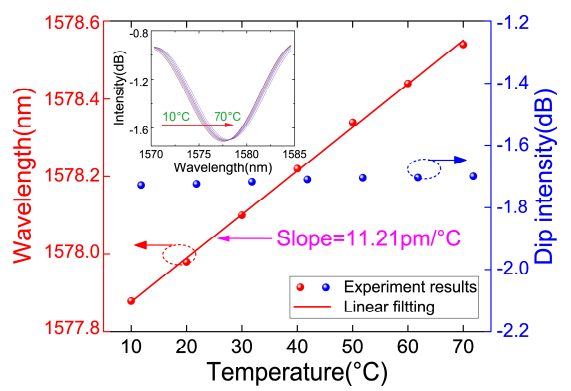

Fig. 6 Linear relationship between the wavelength shift of the interference dip at $\sim 1578 \mathrm{~nm}$ for the sensor and ambient temperature.

fringe is insensitive to temperature. Since the temperature information can be independently decoded from the wavelength shift of the fringe pattern, our proposed RI sensor can achieve simultaneous measurement of RI and temperature, and thus solve the cross-sensitivity problem between temperature and surrounding RI. As the RI of a liquid is usually temperature dependent, the simultaneous measurement is extremely advantageous since the concentration of a solution for chemical sensing is then known. The wavelength of the interference fringe dip of our FPI sensor is completely insensitive to the surrounding RI, so the temperature can be monitored easily.

In conclusions, an in-line fiber intensity modulated RI sensor based on an FPI formed by inducing a refractive-index-modified-dot in a fiber using a fs laser has been demonstrated in this paper. The developed device has very favorable characteristics and numerous advantages. Experimental results show that such a FPI probe sensor exhibits an ultra-high resolution of $4.0 \times 10^{-7} \mathrm{RIU}$ and an ultra-high sensitivity of $2523.2 \mathrm{~dB} / \mathrm{RIU}$ at the refractive index of 1.435, which is at least 10 times higher than the other intensity modulated RI sensors reported previously. Moreover, the probe can also be used to monitor temperature with a sensitivity of $\sim 11.21 \mathrm{pm} /{ }^{\circ} \mathrm{C}$, which allows a simultaneous measurement of the surrounding RI and temperature. Moreover, our theoretical analysis reveals the existence of two ultra-sensitive zones for RI sensing for each device, which is also verified experimentally. To the authors' knowledge, it is the first time that this important phenomenon in FPI sensors is revealed. These excellent features of the device in addition to such merits as ultra-high sensitivity, low cost, a simple and compact structure, high reproducibility, good robust, time-saving in fabrication and the ability to overcome the temperature cross sensitivity make it a very promising candidate in practical environmental, chemical and biochemical sensing applications.

Funding. Director Fund of WNLO; National 1000 Young Talents Program, China; 111 Project (No. B07038)

\section{References}

1. K. Milenko, D. J. Hu, P. P. Shum, T. Zhang, J. L. Lim, Y. Wang, T. R. Wolinski, H. Wei, and W. Tong, Opt.Lett. 37, 1373 (2012).

2. H. Y. Choi, G. Mudhana, K. S. Park, U. C. Paek, and B. H. Lee, Opt. Express 18, 141 (2010).

3. Y. Zhang, X. P. Chen, Y. X. Wang, K. L. Cooper, and A. B. Wang, J. Lightwave Technol. 25, 1797 (2007).

4. D. W. Duan, Y. J. Rao, Y. S. Hou, and T. Zhu, Appl. Opt. 51, 1033 (2012).

5. D. Donlagic and E. Cibula, Opt. Lett. 30, 2071 (2005).

6. C. Li, Q. Liu, X. Peng, and S. Fan, Opt.Express 23, 27494 (2015)

7. Y. J. Rao, M. Deng, D. W. Duan, X. C. Yang, T. Zhu, and G. H. Cheng, Opt.Express 15, 14123 (2007).

8. Y. Liu, S. Qu, and Y. Li, Opt.Lett. 38, 335 (2013).

9. M. Tian, P. Lu, L. Chen, D. Liu, M. Yang, and J. Zhang, Opt.Commun. 316, 80 (2014).

10.Z. L. Ran, Y. J. Rao, W. J. Liu, X. Liao, and K. S. Chiang, Opt.Express 16, 2252 (2008).

11. Y. Kondo, K. Nouchi, T. Mitsuyu, M. Watanabe, P. G. Kazansky, and K. Hirao, Opt.Lett. 24, 646 (1999).

12.J. Zhou, Y. Wang, C. Liao, B. Sun, J. He, G. Yin, S. Liu, Z. Li, G. Wang, X. Zhong, and J. Zhao, Sensor. Actuat.B- Chem 208, 315 (2015).

13. S. Silva, O. Frazão, J. L. Santos, and F. X. Malcata, Sensor. Actuat.B- Chem 161, 88 (2012).

14. S. F. O. Silva, Opt. Eng. 47, 054403 (2008)

15. Y. Ma, X. G. Qiao, T. Guo, R. H. Wang, J. Zhang, Y. Y. Weng, Q. Z. Rong, M. L. Hu, and Z. Y. Feng, Chin.Opt.Lett. 10(2012).

16. Y. Wang, D. N. Wang, M. W. Yang, W. Hong, and P. X. Lu, Opt.Lett. 34, 3328 (2009). 


\section{full references}

1. K. Milenko, D. J. Hu, P. P. Shum, T. Zhang, J. L. Lim, Y. Wang, T. R. Wolinski, H. Wei, and W. Tong, "Photonic crystal fiber tip interferometer for refractive index sensing," Optics letters 37(8), 1373-1375 (2012).

2. H. Y. Choi, G. Mudhana, K. S. Park, U. C. Paek, and B. H. Lee, "Cross-talk free and ultra-compact fiber optic sensor for simultaneous measurement of temperature and refractive index," Optics express 18(1), 141-149 (2010).

3. Y. Zhang, X. P. Chen, Y. X. Wang, K. L. Cooper, and A. B. Wang, "Microgap multicavity Fabry-Perot biosensor," J Lightwave Technol 25(7), 1797-1804 (2007).

4. D. W. Duan, Y. J. Rao, Y. S. Hou, and T. Zhu, "Microbubble based fiber-optic Fabry-Perot interferometer formed by fusion splicing single-mode fibers for strain measurement," Appl Optics 51(8), 1033-1036 (2012).

5. D. Donlagic and E. Cibula, "All-fiber high-sensitivity pressure sensor with SiO2 diaphragm," Optics letters 30(16), 2071-2073 (2005).

6. C. Li, Q. Liu, X. Peng, and S. Fan, "Analyzing the temperature sensitivity of Fabry-Perot sensor using multilayer graphene diaphragm," Optics express 23(21), 27494-27502 (2015).

7. Y. J. Rao, M. Deng, D. W. Duan, X. C. Yang, T. Zhu, and G. H. Cheng, "Micro Fabry-Perot interferometers in silica fibers machined by femtosecond laser," Optics express 15(21), 1412314128 (2007)

8. Y. Liu, S. Qu, and Y. Li, "Single microchannel high-temperature fiber sensor by femtosecond laser-induced water breakdown," Optics letters 38(3), 335-337 (2013).

9. M. Tian, P. Lu, L. Chen, D. Liu, M. Yang, and J. Zhang, "Femtosecond laser fabricated in-line micro multicavity fiber FP interferometers sensor," Optics Communications 316(80-85 (2014).

10. Z. L. Ran, Y. J. Rao, W. J. Liu, X. Liao, and K. S. Chiang, "Lasermicromachined Fabry-Perot optical fiber tip sensor for highresolution temperature-independent measurement of refractive index," Optics express 16(3), 2252-2263 (2008).

11. Y. Kondo, K. Nouchi, T. Mitsuyu, M. Watanabe, P. G. Kazansky, and K. Hirao, "Fabrication of long-period fiber gratings by focused irradiation of infrared femtosecond laser pulses," Optics letters 24(10), 646-648 (1999).

12. J. Zhou, Y. Wang, C. Liao, B. Sun, J. He, G. Yin, S. Liu, Z. Li, G. Wang, X. Zhong, and J. Zhao, "Intensity modulated refractive index sensor based on optical fiber Michelson interferometer," Sensors and Actuators B: Chemical 208(315-319 (2015).

13. S. Silva, O. Frazão, J. L. Santos, and F. X. Malcata, "A reflective optical fiber refractometer based on multimode interference," Sensors and Actuators B: Chemical 161(1), 88-92 (2012).

$14 . \quad$ S. F. O. Silva, "Optical fiber refractometer based on a FabryPérot interferometer," Optical Engineering 47(5), 054403 (2008).

15. Y. Ma, X. G. Qiao, T. Guo, R. H. Wang, J. Zhang, Y. Y. Weng, Q. Z. Rong, M. L. Hu, and Z. Y. Feng, "Temperature-independent refractive index measurement based on Fabry-Perot fiber tip sensor modulated by Fresnel reflection," Chin Opt Lett 10(5)(2012).

16. Y. Wang, D. N. Wang, M. W. Yang, W. Hong, and P. X. Lu, "Refractive index sensor based on a microhole in single-mode fiber created by the use of femtosecond laser micromachining," Optics letters 34(21), 3328-3330 (2009). 